\title{
Toll-like receptor 4 plays a tumor-suppressive role in cutaneous squamous cell carcinoma
}

\author{
ERINA MIKAMI ${ }^{1,2}$, MITSUHIRO KUDO $^{1}$, RYUJI OHASHI $^{3}$, KIYOKO KAWAHARA $^{1}$, \\ YOKO KAWAMOTO $^{1}$, KIYOSHI TEDUKA ${ }^{1}$, TAKENORI FUJII ${ }^{1}$, TAEKO KITAMURA ${ }^{1}$, SHOKO KURE ${ }^{1,4}$, \\ KOUSUKE ISHINO $^{1}$, TAKASHI SAKATANI ${ }^{1,4}$, RYUICHI WADA ${ }^{1,4}$, HIDEHISA SAEKI ${ }^{2}$ and ZENYA NAITO ${ }^{1,4}$ \\ Departments of ${ }^{1}$ Integrated Diagnostic Pathology and ${ }^{2}$ Dermatology, Nippon Medical School, Tokyo 113-8603; \\ ${ }^{3}$ Department of Diagnostic Pathology, Nippon Medical School Musashikosugi Hospital, Kawasaki, Kanagawa 211-8533; \\ ${ }^{4}$ Department of Diagnostic Pathology, Nippon Medical School Hospital, Tokyo 113-8603, Japan
}

Received October 30, 2018; Accepted April 1, 2019

DOI: 10.3892/ijo.2019.4790

\begin{abstract}
Toll-like receptor 4 (TLR4), a key regulator of the innate immune system, is expressed not only in immune cells, but also in a number of cancer cells. A biological role for TLR4 in cutaneous squamous cell carcinoma (SCC), however, is unclear. In this study, we first examined TLR4 expression and localization in cases of SCC, actinic keratosis (AK) and Bowen's disease (BD) by immunohistochemistry. TLR4 expression was significantly higher in the SCC than in the AK or BD tissues. We then determined the TLR4 expression level in vivo, in 3 histological subtypes of SCC. TLR4 expression in poorly differentiated SCC was significantly lower compared with that of the moderately and well-differentiated type. In addition, the CD44 immunoreactivity tended to be high in the cell membrane of poorly differentiated SCC. Of note, poorly differentiated SCC is a risk factor of unfavorable outcomes in affected patients. We then assessed the biological role of TLR4 in HSC-1 and HSC-5 SCC cells and HaCaT human keratinocytes. TLR4 knockdown by transfection with siRNA accelerated HSC-1 and $\mathrm{HaCaT}$ cell migration and invasion compared to the control siRNA-transfected cells. TLR4 knockdown resulted in an increased CD44 expression and in an enhanced filopodia protrusion formation, particularly in HSC-1. On the whole, these results suggest that a reduced TLR4 expression enhances the malignant features in SCC
\end{abstract}

Correspondence to: Dr Mitsuhiro Kudo, Department of Integrated Diagnostic Pathology, Nippon Medical School, 1-1-5 Sendagi, Bunkyo-ku, Tokyo 113-8603, Japan

E-mail:kudoh@nms.ac.jp

Abbreviations: TLR4, Toll-like receptor 4; SCC, squamous cell carcinoma; AK, actinic keratosis; BD, Bowen's disease; FFPE, formalin-fixed paraffin-embedded

Key words: Toll-like receptor 4, cutaneous squamous cell carcinoma, differentiation, CD 44, migration, invasion, filopodia cases and cultured SCC cell lines. TLR4 may thus play an anti-tumor role in cutaneous SCC.

\section{Introduction}

Cutaneous squamous cell carcinoma (SCC) is the second most frequent skin cancer after basal cell carcinoma, and the second leading cause of skin cancer-associated mortality after malignant melanoma $(1,2)$. Although the majority of SCC tumors are resected at an early stage, SCC carries a risk of local recurrence and lymph node metastasis. Investigations into molecules associated with the unfavorable phenotypes of cancer are important for the diagnosis and effective treatment of cutaneous SCC.

Toll-like receptor 4 (TLR4) is a transmembrane protein and member of the Toll-like receptor family. TLR4 is well known as a key regulator of innate immunity; however, its perturbed expression has also been observed in a number of types of cancer, including ovarian cancer (3), pancreatic cancer (4), hepatocellular carcinoma (5), colorectal cancer $(6,7)$, malignant melanoma (8) and skin cancers (9-11). TLR4 may exert anti-tumor or pro-tumor effects, depending on the tumor type and whether it is expressed in tumor cells or immune cells (12-14). TLR4 is expressed in both normal and pathologic skin cells, and is involved in several skin diseases, including skin cancers (15-17). Recent studies have demonstrated that the TLR4 antagonist, resatorvid, blocks solar UV-induced skin tumorigenesis in mice ex vivo and in vivo $(9,10)$. These studies have indicated that TLR4 may exert pro-tumor effects and may thus be a suitable target with which to prevent photo-carcinogenesis. Anti-tumor effects of TLR4 in cutaneous $\mathrm{SCC}$, however, have also been reported $(11,18)$. In a previous study, TLR4 knockdown by small hairpin RNA (shTLR4) was shown to induce HaCaT keratinocyte proliferation in vitro. Conversely, the overexpression of TLR4 in the SCC13 cell line reduced proliferation compared to TLR4-negative SCC13 cells in vitro. Moreover, the growth rate of TLR4-overexpressing SCC13 tumors was attenuated compared to TLR4-negative SCC13 tumors in a xenograft mouse model (11). These findings indicate the possibility for an anti-tumor role of TLR4 in cutaneous SCC. However, 
whether TLR4 is truly an anti-tumor or pro-tumor molecule in cutaneous SCC remains to be determined.

CD44 is a transmembrane glycoprotein that is expressed in different variant forms in several types of cancer, including cutaneous SCC (19-21). CD44 interacts with extracellular matrix ligands to regulate cell-matrix and cell-cell interactions, and to promote metastasis (22). Hyaluronan-activated CD44 promotes RhoGTPase signaling, leading to keratinocyte activities, such as cell adhesion, proliferation and migration (23). A hyaluronan-mediated CD44 interaction with TLR4 has been demonstrated in NDA-MB-123 breast cancer cells (24); however, the interaction between CD44 and TLR4 in skin cancers remains unknown.

In this study, we examined the biological role of TLR4 in cutaneous SCC. We confirmed the expression and localization of TLR4 in non-melanocytic skin cancer tissues by immunohistochemistry and analyzed the biological effects of TLR4 and the expression of CD44 on cutaneous SCC in vitro.

\section{Materials and methods}

Formalin-fixed paraffin-embedded (FFPE) tissue samples. A total of 36 skin tumor, $5 \mathrm{AK}, 5 \mathrm{BD}$ and $26 \mathrm{SCC}$ cases were obtained from the Nippon Medical School Hospital archives (Tokyo, Japan). The AK and BD cases were obtained between 2015 and 2017. The SCC cases were obtained between 2009 and 2015. This study was carried out in accordance with the Declaration of Helsinki, 2013, and the Japanese Society of Pathology Ethics Committee. The Nippon Medical School Hospital Institutional Review Board approved this study (approval no. 29-07-788, August 18th, 2017) and written informed consent was obtained from all patients. All cases were carefully reviewed, and pathological diagnoses were made according to the WHO classification (25). SCC cases were classified as well-differentiated $(>75 \%)$, moderately differentiated $(25-75 \%)$, or poorly differentiated $(<25 \%)$ tumors based on the degree of keratinization. The clinicopathological data of the SCC cases are presented in Table I.

The AK cases included 3 males and 2 females, and the age of the patients ranged from 71 to 91 years, with a mean age of 82.2 years. The lesion locations were all found on the face $(5 / 5)$. The BD cases included 3 males and 2 females, and the age of the patients ranged from 62 to 89 years, with a mean age of 74.2 years. The lesion locations included 4 on the extremities (4/5) and 1 on the trunk (1/5).

Immunohistochemistry. The FFPE tissue sections were stained for TLR4 or CD44. Following deparaffinization, the sections were pre-treated in an autoclave at $121^{\circ} \mathrm{C}$ for $15 \mathrm{~min}$ in $10 \mathrm{mM}$ citrate buffer ( $\mathrm{pH}$ 6.0). Endogenous peroxidase was blocked using $0.3 \%$ hydrogen peroxide in methanol for $30 \mathrm{~min}$. The sections were then incubated with an anti-TLR4 mouse monoclonal antibody (1:100; ab89455; Abcam, Cambridge, UK) or an anti-human CD44 monoclonal mouse antibody (1:10,000; BBA10; R\&D Systems, Inc. Minneapolis, MN, USA) in phosphate-buffered saline containing $1 \%$ bovine serum albumin at $4^{\circ} \mathrm{C}$ overnight. The sections were further incubated with Simple Stain MAX-PO (M; Nichirei Biosciences Inc., Tokyo, Japan) for $40 \mathrm{~min}$ and peroxidase activity was visualized by $0.02 \%$ diaminobenzidine containing $0.003 \%$ hydrogen peroxide for $2 \mathrm{~min}$. The sections were then counterstained with Mayer's hematoxylin. We could not collect normal skin or other tissues as control tissues. However, normal epidermis and skin appendage adjacent to the tumors were consistently positive for TLR4; in addition, normal epidermis and the secretory part of the sweat gland adjacent to the tumors were consistently positive for CD44 in all of the cases. Thus, the normal epidermis and skin appendage served as an internal positive control for TLR4 staining, and the normal epidermis and sweat gland served as an internal positive control for CD44 staining. Tissues stained without primary antibody were used as negative controls in each staining.

Evaluation of the results of immunohistochemistry. TLR4-stained slides were scanned at x40 magnification and digitized using a Leica SCN400 slide scanner (Leica Microsystems, Wetzlar, Germany). The acquired images were analyzed using HistoQuest cell analysis software 4.0 (TissueGnostics, Vienna, Austria) for automated measurements of TLR4 expression intensity and to calculate the percentage of TLR4-positive cells within each slide. Five, randomly selected fields of view were analyzed.

To assess the TLR4 expression levels, the TLR4 integrated intensity was calculated by multiplying the intensity of TLR4-stained cells by the percentage of TLR4-stained cells. This threshold was used for all samples.

Cells and cell culture. Human skin SCC cell lines, HSC-1 (cat. no. JCRB1015) (26) and HSC-5 (cat. no. JCRB1016) (27), were obtained from the Japanese Collection of Research Bioresources (Osaka, Japan). An immortalized human keratinocyte cell line, HaCaT (cat. no. 300493), was purchased from CLS Cell Lines Service GmbH (Eppelheim, Germany). The HSC-1, HSC-5 and HaCaT cells were grown in RPMI-1640 (Gibco; Thermo Fisher Scientific, Inc., Waltham, MA, USA) medium containing $10 \%$ fetal bovine serum (FBS; Nichirei Biosciences Inc., Tokyo, Japan) at $37^{\circ} \mathrm{C}$ in a humidified $5 \% \mathrm{CO}_{2}$ atmosphere.

Knockdown of TLR4 expression in HSC-1, HSC-5 and HaCaT cells. The HSC-1, HSC-5 and HaCaT cells were transfected using Lipofectamine ${ }^{\circledR}$ RNAiMAX Reagent (Invitrogen; Thermo Fisher Scientific, Inc.) with $5 \mathrm{nM}$ pre-designed TLR4 siRNA (siTLR4; no. 4390824; Ambion; Thermo Fisher Scientific, Inc.), or $5 \mathrm{nM}$ negative control siRNA (siCtrl; no. 4390844; Ambion; Thermo Fisher Scientific, Inc.) according to the manufacturer's instructions.

\section{Reverse transcription-quantitative polymerase chain reaction} $(R T-q P C R)$. A total of $2.5 \times 10^{5}$ cells were seeded in $60-\mathrm{mm}$ dishes and cultured for $48 \mathrm{~h}$ at $37^{\circ} \mathrm{C}$ in a humidified $5 \% \mathrm{CO}_{2}$ atmosphere. Total RNA was extracted using a FastPure RNA kit, and $1 \mu \mathrm{g}$ total RNA was used for reverse transcription using a SuperScript VILO cDNA Synthesis kit, as per the manufacturer's instructions (Thermo Fisher Scientific, Inc.). RT-qPCR for TLR4, CD44 and 18S rRNA (as an internal standard) was performed using a StepOnePlus Real-Time PCR system (Thermo Fisher Scientific, Inc.) with specific primers (18S: Hs 03928990_g1, TLR4: Hs 00152939_m7, CD44: Hs 00153304_m7, Thermo Fisher Scientific, Inc.) and a TaqMan 
Table I. Clinicopathological data of SCC cases.

\begin{tabular}{|c|c|c|c|c|c|c|c|}
\hline Case & Age (years) & Sex & Clinical stage & Location & Size (mm) & Depth of invasion (mm) & Differentiation \\
\hline 1 & 86 & M & I & Face & 17 & 7.5 & Well \\
\hline 2 & 83 & M & II & Face & 30 & 7.1 & Well \\
\hline 3 & 53 & $\mathrm{M}$ & $\mathrm{I}$ & Head & 11 & 5.9 & Well \\
\hline 4 & 70 & $\mathrm{M}$ & $\mathrm{I}$ & Lip & 6 & 2.9 & Poor \\
\hline 5 & 77 & $\mathrm{~F}$ & II & Face & 23 & 1.2 & Poor \\
\hline 6 & 73 & M & $\mathrm{I}$ & Face & 10 & 1.2 & Mod \\
\hline 7 & 69 & $\mathrm{~F}$ & II & Lip & 30 & 4.1 & Mod \\
\hline 8 & 92 & $\mathrm{~F}$ & I & Face & 14 & 3.5 & Mod \\
\hline 9 & 71 & M & I & Extremities (finger) & 10 & 2.2 & Mod \\
\hline 10 & 89 & $\mathrm{M}$ & III & Head & 49 & 9.6 & Well \\
\hline 11 & 76 & $\mathrm{~F}$ & $\mathrm{I}$ & Face & 8 & 0.8 & Well \\
\hline 12 & 86 & M & II & Head & 20 & 7.2 & Poor \\
\hline 13 & 70 & M & II & Extremities (foot) & 25 & 9.3 & Mod \\
\hline 14 & 92 & $\mathrm{~F}$ & II & Face & 20 & 2.9 & Mod \\
\hline 15 & 66 & $\mathrm{M}$ & $\mathrm{I}$ & Genital Area & 9 & 1.6 & Poor \\
\hline 16 & 72 & $\mathrm{~F}$ & $\mathrm{I}$ & Extremities (finger) & 8 & 3.5 & Mod \\
\hline 17 & 57 & M & III & Genital Area & 11 & 3.3 & Mod \\
\hline 18 & 82 & $\mathrm{~F}$ & III & Genital Area & 60 & 6.9 & Mod \\
\hline 19 & 95 & $\mathrm{~F}$ & II & Trunk (abdomen) & 26 & 2.2 & Mod \\
\hline 20 & 88 & M & $\mathrm{I}$ & Face & 13 & 3.3 & Mod \\
\hline 21 & 92 & $\mathrm{~F}$ & $\mathrm{I}$ & Face & 19 & 1.2 & Poor \\
\hline 22 & 89 & $\mathrm{~F}$ & II & Face & 30 & 1.2 & Poor \\
\hline 23 & 87 & $\mathrm{~F}$ & II & Face & 30 & 1.7 & Mod \\
\hline 24 & 89 & M & I & Face & 13 & 1.6 & Mod \\
\hline 25 & 79 & $\mathrm{~F}$ & I & Head & 16 & 6.1 & Well \\
\hline 26 & 65 & M & I & Extremities (hand) & 8 & 2.3 & Mod \\
\hline
\end{tabular}

SCC, squamous cell carcinoma; Clinical stage, TNM Classification of malignant tumors, 8th edition (36). Well, well-differentiated; Mod, moderately differentiated; Poor, poorly differentiated.

probe (Thermo Fisher Scientific, Inc.). The cycling conditions were as follows: $20 \mathrm{sec}$ at $95^{\circ} \mathrm{C}$, and then 40 cycles of $1 \mathrm{sec}$ at $95^{\circ} \mathrm{C}$, and $20 \mathrm{sec}$ at $60^{\circ} \mathrm{C}$. The RT-qPCR results are expressed as the ratio of target $\mathrm{mRNA}$ to $18 \mathrm{~S}$ rRNA and analyzed using the $\Delta \Delta \mathrm{Cq}$ method (28).

Western blot analysis. Total proteins were extracted from the cells using 10X Cell Lysis Buffer (no. 9803; Cell Signaling Technology, Danvers, MA, USA) according to the manufacturer's instructions. The supernatants were collected as a cell extract, and the protein concentration was measured by Pierce 660 nm Protein Assay (Thermo Fisher Scientific, Inc.). Equal amounts of protein $(10 \mu \mathrm{g})$ for each cell extract were loaded onto 5-20\% sodium dodecyl sulfate-polyacrylamide gels and separated by electrophoresis (SDS-PAGE) and then electrophoretically blotted onto a polyvinylidene difluoride (PVDF) membrane (Trans-Blot Turbo Mini PVDF Transfer Packs, Bio-Rad Laboratories, Inc., Richmond, CA, USA). The blots were blocked for 30 min with 5\% skimmed milk in Tris-buffered saline (TBS) containing $0.01 \mathrm{M}$ Tris- $\mathrm{HCl}$, $150 \mathrm{mM} \mathrm{NaCl}$ and $0.05 \%$ Tween-20, and then incubated with an anti-human TLR4 antibody (1:500; ab89455; Abcam) and an anti-human CD44 monoclonal mouse antibody (1:1,000; no. 3570; Cell Signaling Technology), or anti- $\beta$-actin monoclonal mouse antibody (1:10,000; Clone AC-74; Sigma-Aldrich, St. Louis, MO, USA) overnight at $4^{\circ} \mathrm{C}$. After 30 -min washing in TBS with $0.01 \%$ Triton $\mathrm{X}-100$, the blots were incubated with a horseradish peroxidase-conjugated secondary antibody (1:10,000, A106PU; American Qualex Antibodies, San Clemente, CA, USA) for $1 \mathrm{~h}$ at room temperature. Signals were visualized using a Clarity Max Western ECL Substrate (no. 1705062; Bio-Rad Laboratories, Inc.) for TLR4 and CD44, and a Super Signal West Pico Chemiluminescence substrate (Thermo Fisher Scientific, Inc.) for $\beta$-actin. Immunoreactive bands were quantified using Fiji-ImageJ software version 2.0.0 (https://imagej.nih.gov/ij/). Experiments were performed in triplicate.

Cell migration and invasion assays. In vitro migration and invasion assays were carried out by Boyden chamber assay using BioCoat control inserts and BioCoat Matrigel-coated inserts with BioCoat chambers (BD Biosciences, San Jose, CA, USA). Following transfection with the siRNA for $72 \mathrm{~h}$, the cells were harvested and suspended in serum-free RPMI-1640. 
A
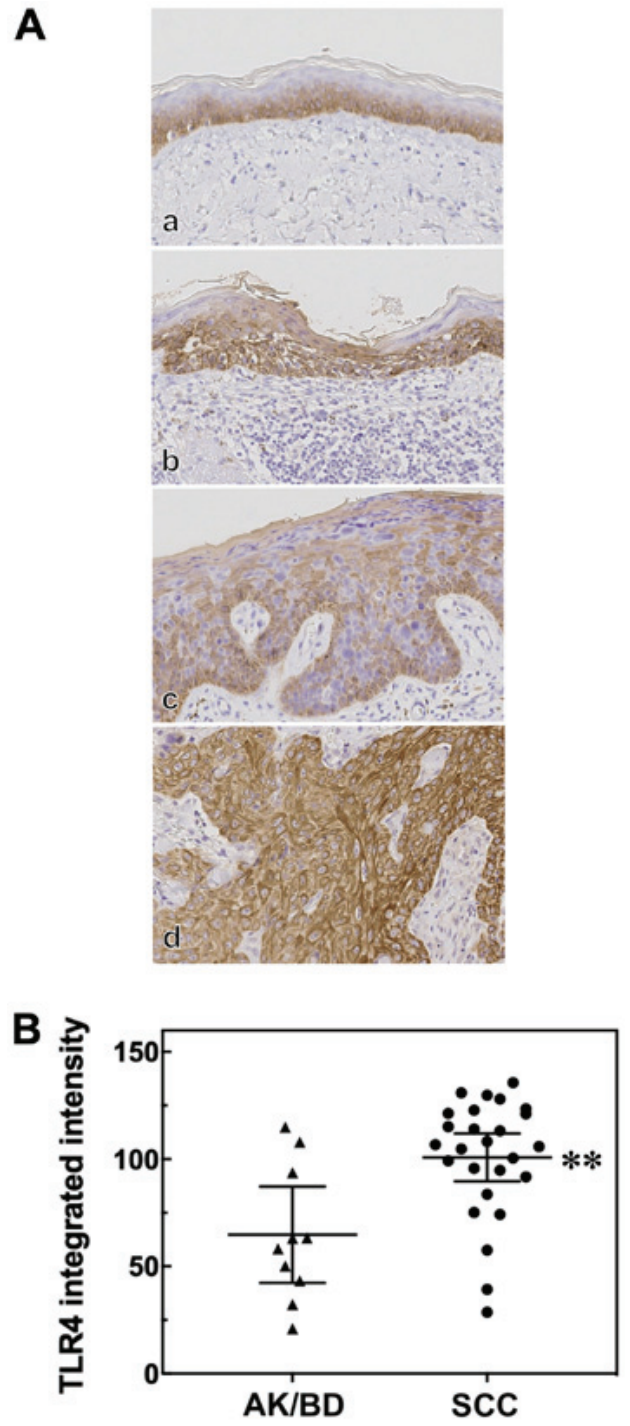

Figure 1. TLR4 expression in the peritumoral region, AK, BD and SCC. (A) Sections were stained with a TLR4 antibody. (Panel a) TLR4 expression in the peritumoral region was confined to one or more layers in the basal epidermis. All cases of (panel b) AK, (panel c) BD, and (panel d) SCC were positive for TLR4. Original magnification, x200. (B) The TLR4 integrated intensity score of the SCC case group (SCC) was significantly higher than the group that combined $\mathrm{AK}$ and $\mathrm{BD}(\mathrm{AK} / \mathrm{BD})$. The data represent the means $\pm 95 \%$ confidence interval. Statistical significance was determined by Mann-Whitney U test $\left({ }^{* *} \mathrm{P}=0.0048\right)$ compared to AK/BD. TLR4, Toll-like receptor 4; SCC, squamous cell carcinoma; AK, actinic keratosis; $\mathrm{BD}$, Bowen's disease.

The cells were then applied to the surface of control or Matrigel-coated inserts at a density of $1 \times 10^{5}$ cells per insert, and culture medium with 10\% FBS was added to the lower chamber to serve as chemoattractant. The cells were incubated for $24 \mathrm{~h}$ (HSC- 1 and $\mathrm{HSC}-5$ cells) or $36 \mathrm{~h}$ (HaCaT cells) at $37^{\circ} \mathrm{C}$ in a humidified $5 \% \mathrm{CO}_{2}$ atmosphere, before the migrating and invading cells were stained with Diff-Quick ${ }^{\mathrm{TM}}$ three-step stain kit (Sysmex Corp., Kobe, Japan). Stained cells on the outer surface in 5 randomly selected fields per insert were counted under a bright field microscope (Olympus, Tokyo, Japan) with a X20 objective. Experiments were performed in triplicate.

Immunofluorescence staining. The HSC-1, HSC-5 and $\mathrm{HaCaT}$ cells were seeded in $35-\mathrm{mm}$ glass bottomed dishes at
A
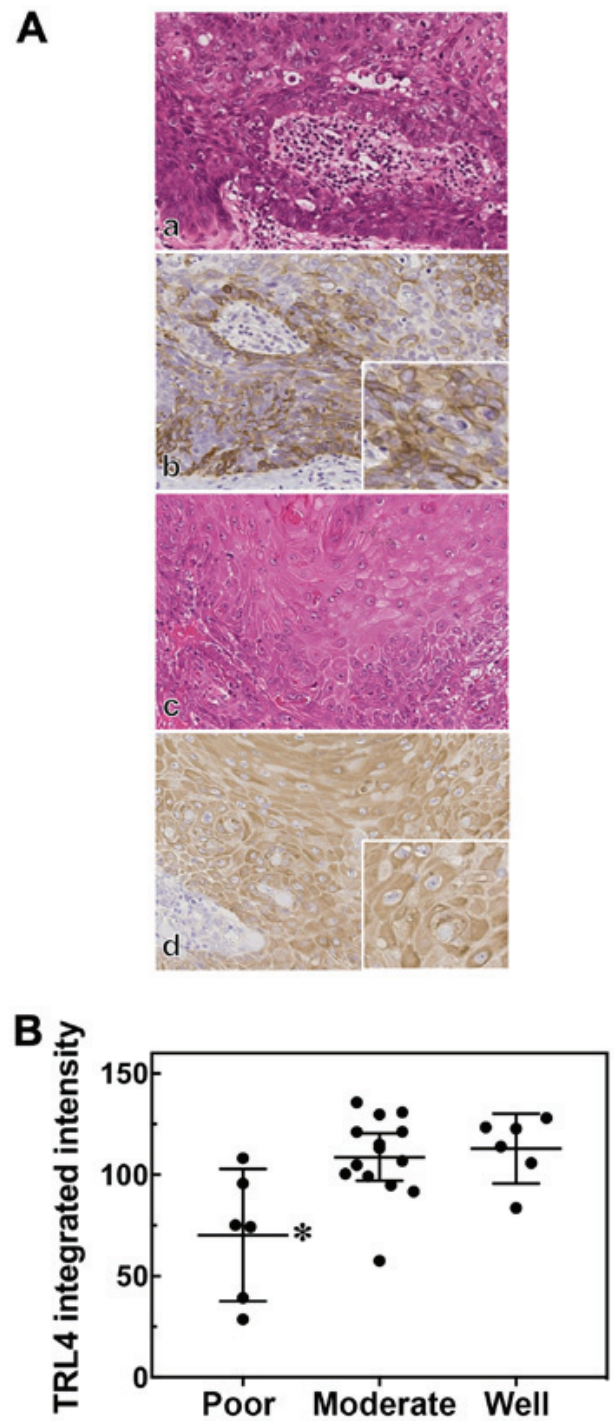

Figure 2. Differences in TLR4 expression are dependent on the degree of SCC differentiation. (A) Poorly differentiated SCC and well-differentiated SCC were labeled with (panels a and c) hematoxylin and eosin (H\&E) and (panels $b$ and d) stained with a TLR4 antibody. (Panel b) TLR4 expression in poorly differentiated SCC was sporadic and localized to the cell membrane. The inset in (panel b) shows a high-power view. (Panel d) TLR4 expression in well-differentiated SCC was diffuse and homogeneous. The inset in (panel d) shows a high-power view. Original magnification, x200. (B) The TLR4 integrated intensity score was significantly decreased in poorly differentiated SCC compared to moderately differentiated and well-differentiated SCC. The data represent the means $\pm 95 \%$ confidence interval. Statistical significance was determined by Kruskal-Wallis test followed by Dunn's multiple comparison test. Poor vs. moderate, ${ }^{*} \mathrm{P}=0.0407$; poor vs. well differentiated, ${ }^{*} \mathrm{P}=0.0382$. TLR4, Toll-like receptor 4; SCC, squamous cell carcinoma.

$2.0 \times 10^{4}$ cells/dish and incubated with the relevant siRNAs for $72 \mathrm{~h}$. The cells were then fixed with $4 \%$ paraformaldehyde/PBS for $15 \mathrm{~min}$ and then incubated with an anti-TLR4 antibody $(1: 100)$ or anti-CD44 antibody $(1: 400)$ at $4^{\circ} \mathrm{C}$ overnight. The cells were washed with PBS, and then incubated with Alexa 488 (1:1,000; A11001; Invitrogen; Thermo Fisher Scientific, Inc.) or Alexa 568 conjugated secondary antibodies $(1: 1,000$; A11031; Invitrogen; Thermo Fisher Scientific, Inc.) for $1 \mathrm{~h}$ in the dark at room temperature. Following incubation, the cells were washed with PBS and mounted with Vectashield H-1200 containing DAPI (Vector Laboratories, Inc., Burlingame, CA, USA). Tissues stained without primary antibody were 


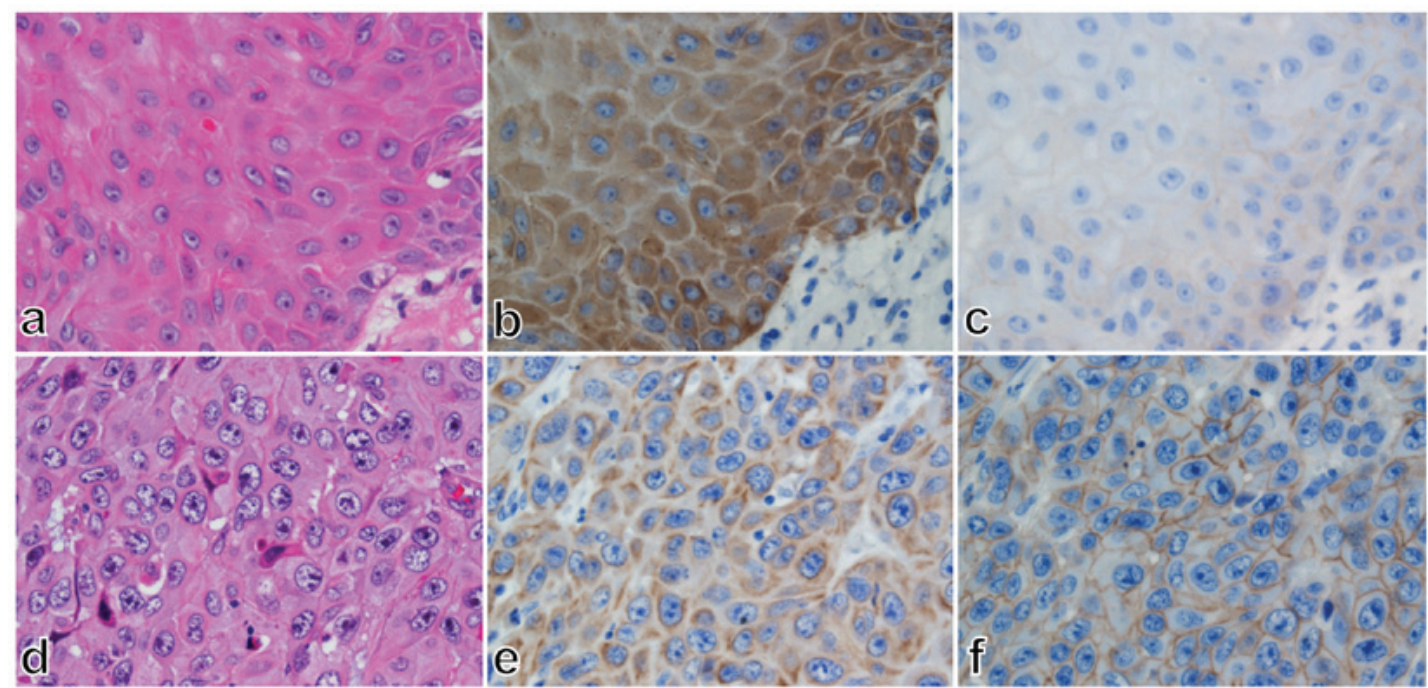

Figure 3. TLR4 and CD44 expression in well and poorly differentiated SCC. Representative images of (panels b and e) TLR4 staining and (panels c and f) CD44 staining of adjacent sections in (panels a-c) well-differentiated SCC and (panels d-f) poorly differentiated SCC. Original magnification, x600. TLR4, Toll-like receptor 4; SCC, squamous cell carcinoma.

used as negative controls in each staining. The fluorescent images were observed under a Digital Eclipse C1 TE2000-E confocal microscope (Nikon Insteck Co., Ltd., Tokyo, Japan) and analyzed using Digital Eclipse $\mathrm{C} 1$ control software EZ-C1 (Version 3.8; Nikon Insteck Co., Ltd.). For imaging analysis, the confocal settings such as the laser intensity and detector sensitivity were unchanged during the acquisition of all images (X20 objective). The analysis of integrated density related to the fluorescence signal of TLR4 and CD44 was carried out on 5 randomly selected fields using Fiji-ImageJ software version 2.0.0 (https://imagej.nih.gov/ij/). The total cell number in each field was counted visually. The quantitative evaluation of the fluorescence signal was carried out by dividing the total integrated density by the total cell number of each field.

Statistical analysis. The data represent the means $\pm 95 \%$ confidence interval or the means \pm standard error of the mean (SEM). A Mann-Whitney U-test, two-way ANOVA Kruskal-Wallis test followed by Dunn's multiple comparison test, two-way ANOVA followed by Sidak's multiple comparisons test, or the multiple test using the Holm-Sidak method were used to evaluate statistical significance. A value of $\mathrm{P}<0.05$ was considered to indicate a statistically significant difference. All statistical analyses were performed using GraphPad Prism version 7 (GraphPad Software, Inc., La Jolla, CA, USA).

\section{Results}

TLR4 expression is elevated in SCC tissues compared to AK and $B D$. We first analyzed TLR4 expression in AK, BD and SCC patient tissues by immunohistochemistry (Fig. 1A). We found that the TLR4-positive cells in the normal epidermis adjacent to the tumors were confined to one or more layers of the basal epidermis (Fig. 1A, panel a). The AK, BD and SCC lesions were all TLR4-positive (Fig. 1A, panels b-d, respectively). In AK and BD, TLR4 immunoreactivity was diffusely distributed in the cytoplasm. TLR4 expression in the skin tumors was estimated by calculating the TLR4 integrated intensity. In this study, the number of cases of AK and BD was only 5 cases each, and the TLR4 integrated intensity score and TLR4 expression pattern between AK and BD did not differ significantly. Thus, we compared the SCC group with the AK/BD group. We found that the TLR4 integrated intensity in SCC was significantly higher than that in the combined group of AK or BD cases (Fig. 1B).

TLR4 expression varies depending on the SCC differentiation level. We then assessed TLR4 expression according to 3 levels of SCC differentiation: Poor, moderate and well. The TLR4 immunoreactivity pattern varied among the SCC cases. The poorly differentiated SCC samples exhibited atypical epithelial neoplastic cells without evident keratinization (Fig. 2A, panel a). In poorly differentiated SCC, TLR4 immunoreactivity was sporadic and localized mainly to the cell membrane (Fig. 2A, panel b, inset). On the other hand, TLR4 immunoreactivity was diffusely distributed in the cytoplasm in well-differentiated SCC (Fig. 2A, panels c and d). Furthermore, the TLR4 integrated intensity score in the poorly differentiated SCC cases was significantly lower than that in the moderately differentiated or well-differentiated SCC cases (Fig. 2B).

CD44 expression in well- and poorly differentiated SCC. In addition, we assessed CD44 immunoreactivity in some cases of well- and poorly differentiated SCC. CD44 immunoreactivity tended to be low in the well-differentiated SCC, whose TLR4 immunoreactivity was diffusely distributed in the cytoplasm (Fig. 3, panels a-c). Conversely, CD44 immunoreactivity tended to be high and localized to the cell membrane in poorly differentiated SCC, whose TLR4 immunoreactivity was low and localized to the cell membrane (Fig. 3, panels d-f).

TLR4 MRNA and protein expression in siTLR4-transfected cells. We used 2 human cutaneous SCC cell lines, HSC-1 and HSC-5, and the immortalized human keratinocyte cell line, $\mathrm{HaCaT}$, to assess the TLR4 mRNA and protein expression levels. We found that TLR4 mRNA relative expression in the 
HSC-1 cells was $\sim 12$-fold higher compared with the control siRNA-transfected HaCaT cells. We then knocked down TLR4 using siRNA in the HSC-1, HSC-5 and HaCaT cells (Fig. 4A) and verified the decreased TLR4 expression at the mRNA level. TLR4 protein expression was also successfully decreased in the TLR4 siRNA-transfected HSC-1 and HaCaT cells. However, in the transfected HSC-5 cells, the degree of knockdown appeared to be low, and no significant difference was observed (Fig. 4B).

TLR4 knockdown enhances the migration and invasion of SCC cells. We then assessed the effects of TLR4 on cell migration and invasion by Boyden chamber assay. We found that transfection with TLR4 siRNA enhanced the migration of the HSC-1, HSC-5 and HaCaT cells compared to the control siRNA-transfected cells (Fig. 5A), and enhanced the invasion of the HSC-1 and HaCaT cells compared to the control siRNA-transfected cells (Fig. 5B). The HSC-1 cells exhibited the most prominent response in terms of migration and invasion following TLR4 knockdown compared to the HSC-5 and $\mathrm{HaCaT}$ cells.

TLR4 knockdown enhances CD44 mRNA and protein expression. We then found that the decreased TLR4 expression enhanced CD44 mRNA relative expression in the HSC-1, HSC-5 and HaCaT cells (Fig. 6A). We also detected the increased expression of the CD44 80-kDa isoform (CD44s) in TLR4 siRNA-transfected treated HSC-1 and $\mathrm{HaCaT}$ cells (Fig. 6B). In addition, we detected a $140-\mathrm{kDa}$ CD44 variant (CD44v) in the HSC-5 and $\mathrm{HaCaT}$ cells; CD44v expression was also enhanced upon TLR4 siRNA transfection (Fig. 6B).

Reduced TLR4 expression enhances CD44 expression at the cell membrane. Finally, we performed immunofluorescence to monitor TLR4 and CD44 cellular localization by confocal microscopy. We found that TLR4 (green) was diffusely expressed in the cytoplasm and cell membrane in all cell types (Fig. 7A, panels a-c). Upon transfection with TLR4 siRNA, TLR4 expression in the cytoplasm was decreased, particularly in the HSC-1 cells compared to the siRNA control-transfected cells; TLR4 expression in the cell membrane seemed unaffected (Fig. 7A, panels d-f). In the quantitative evaluation, TLR4 expression was significantly decreased in the TLR4 siRNA-transfected HSC-1 and HaCaT cells (Fig. 8A) Moreover, the TLR4 siRNA-transfected cells exhibited an increased number of filopodia protrusions and TLR4 expression in the cell membrane extended into these protrusions (Fig. 7A, panels d-f, arrowhead). We then detected that CD44 was mainly localized to the cell membrane in all cell types (Fig. 7B, panels a-c). Transfection with TLR4 siRNA increased CD44 expression and enhanced filopodia protrusions compared to the control siRNA-transfected cells (Fig. 7B, panels d-f). All images are single optical sections, but not compressed stacks, and we selected a slice with the most obvious filopodia protrusions. Therefore, there was a height difference between the protrusions and nuclei (blue) in some cells. In the quantitative evaluation, CD44 expression was significantly increased in the TLR4 siRNA-transfected HSC-1 cells (Fig. 8B).
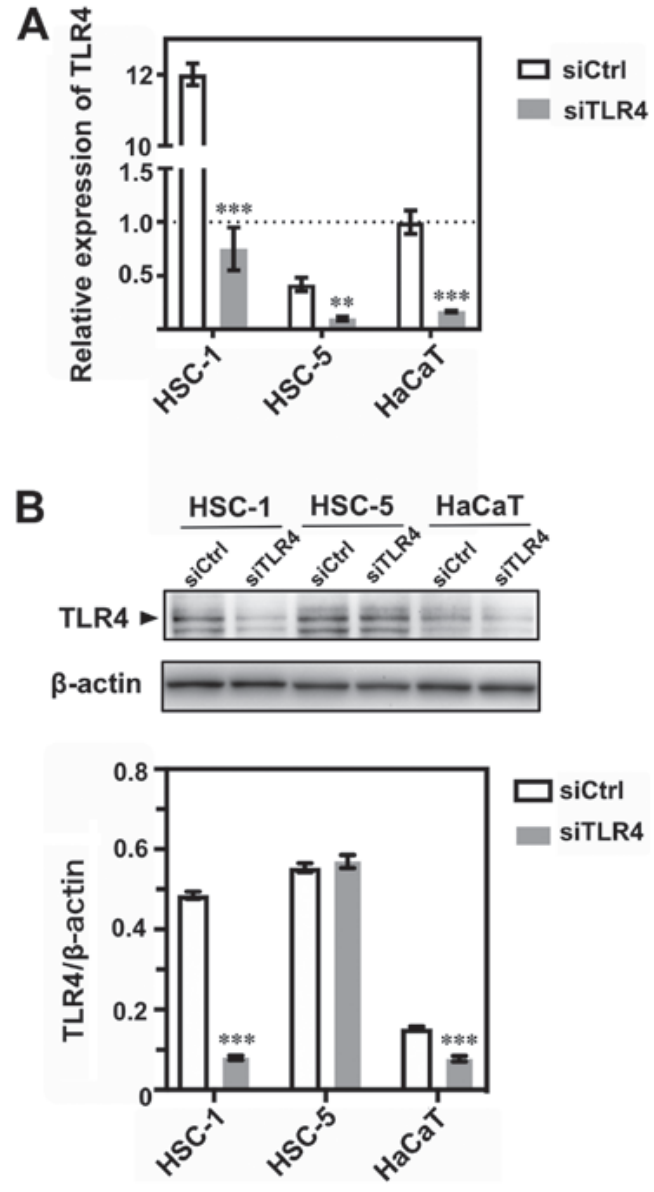

Figure 4. TLR4 mRNA and protein expression in siTLR4-transfected cells (A) Relative expression of TLR4 mRNA was analyzed by RT-qPCR following transfection with siTLR4 in HSC-1, HSC-5 and HaCaT cell lines. The relative expression of TLR4 mRNA is indicated as a percentage of that of siCtrl-transfected HaCaT cells. The data represent the means $\pm 95 \%$ confidence interval of triplicate measurements in 3 independent experiments. Statistical significance between siTLR4 or siCtrl groups and among cell lines was determined by two-way ANOVA followed by Sidak's multiple comparisons test. HSC $-1,{ }^{* * * *} \mathrm{P}<0.001$; HSC $-5,{ }^{* *} \mathrm{P}=0.0029$; HaCaT, ${ }^{* * * *} \mathrm{P}<0.001$. (B) Western blot analysis of TLR4 protein expression following transfection with siTLR4 or siCtrl in HSC-1, HSC-5 and HaCaT cells. The data represent the means $\pm 95 \%$ confidence interval of triplicate measurements in 3 independent experiments. Statistical significance between siTLR4 or siCtrl groups and among cell lines was determined by two-way ANOVA followed by Sidak's multiple comparisons test. HSC-1: ${ }^{* * *} \mathrm{P}<0.001$; HSC-5: $\mathrm{P}=0.1719$; HaCaT: ${ }^{* * *} \mathrm{P}<0.001$. TLR4, Toll-like receptor 4; SCC, squamous cell carcinoma; siTLR4, siRNA against TLR4; siCtrl, control siRNA.

\section{Discussion}

This study examined the expression and localization of TLR4 in non-melanocytic skin cancers AK, BD and SCC. We determined the biological role of TLR4 in SCC in the HSC-1 and HSC-5 SCC cells and HaCaT human keratinocytes. We first quantitatively evaluated TLR4 expression in AK, BD and SCC using the pathological tissue samples. TLR4 was expressed not only in the basal epidermis of normal skin, adjacent to the tumor, but also in all tumor lesions (Fig. 1A). The TLR4 integrated intensity score of SCC group was significantly higher than that of the combined AK/BD group (Fig. 1B).

$\mathrm{AK}$ is considered as a pre-cancerous SCC lesion, and BD is essentially equivalent to and used interchangeably with the term SCC in situ. These lesions both progress and evolve to 

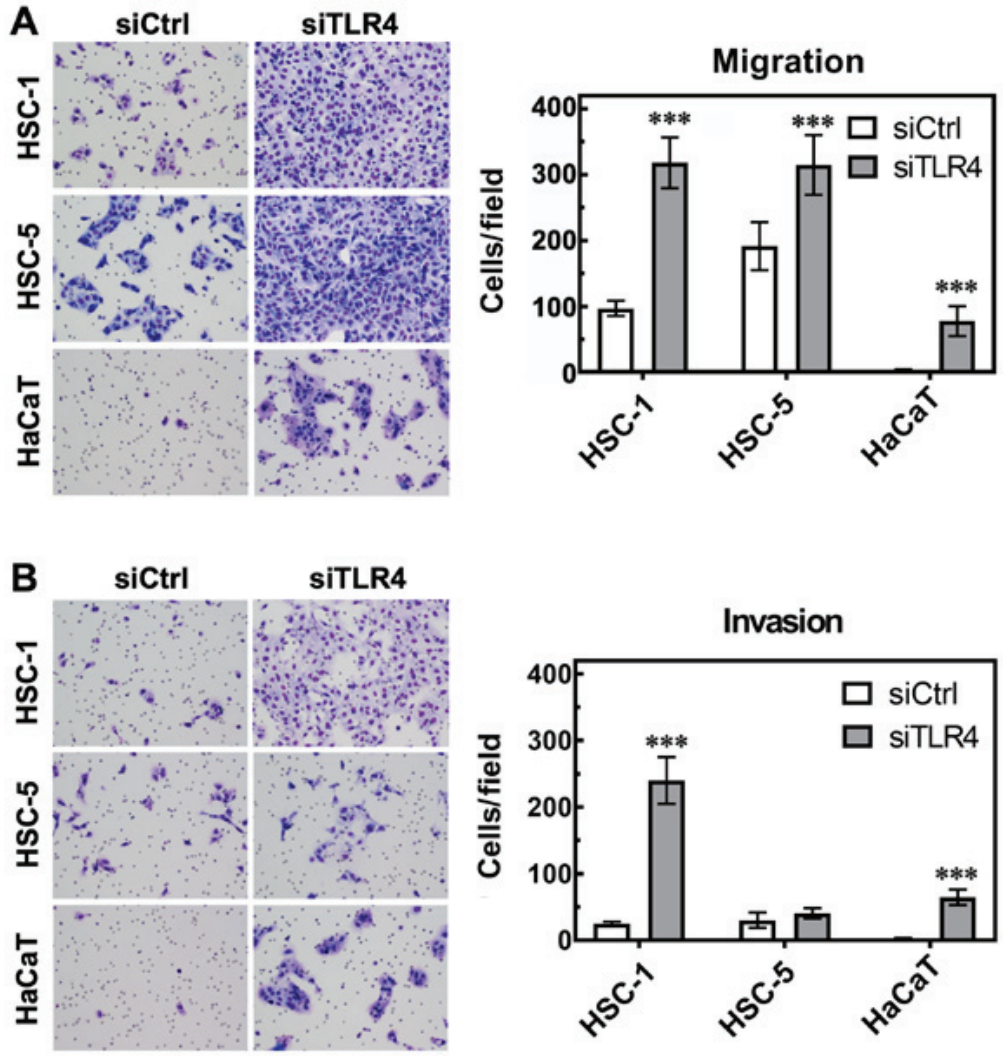

Figure 5. TLR4 knockdown enhances the migration and invasion in SCC and HaCaT cells. The effect of TLR4 on cell migration and cell invasion was evaluated by Boyden chamber assay. (A) TLR4 knockdown enhanced the migration of HSC-1, HSC-5 and HaCaT cell lines. ${ }^{* * *} \mathrm{P}<0.001$. (B) TLR4 knockdown enhanced the invasion of HSC-1 and HaCaT cells. ${ }^{* * *} \mathrm{P}<0.001$. The data represent the means $\pm 95 \%$ confidence interval of triplicate measurements in 3 independent experiments. Statistical significance between siTLR4 or siCtrl groups and among cell lines was determined by two-way ANOVA followed by Sidak's multiple comparisons test. Diff-Quick-stained cells represent in the left panel of A and B. Original magnification, x200. TLR4, Toll-like receptor 4; SCC, squamous cell carcinoma; siTLR4, siRNA against TLR4; siCtrl, control siRNA.

give rise to invasive SCC (29). A previous study reported a significant increase in TLR4 expression in keratinocytes in FFPE tissue samples during the progression from normal skin to AK; the study also detected this increase in expression at a later stage of SCC progression in tissue microarray samples (9). These results indicate that TLR4 expression may be involved in cutaneous SCC formation and progression.

Subsequently, focusing on the TLR4 expression pattern in SCC, we found an association between the SCC differentiation degree and TLR4 expression levels. In poorly differentiated SCC, the TLR4 integrated intensity score was significantly lower than in moderately differentiated or well-differentiated SCC cases (Fig. 2B). We also found that TLR4 immunoreactivity was largely localized to the cell membrane in poorly differentiated SCC (Fig. 2A, panel b, inset). In addition, the CD44 immunoreactivity tended to be high and localized to the cell membrane in poorly differentiated SCC (Fig. 3). Other histological features, such as the site of location, tumor size, or depth of invasion were not associated with TLR4 expression (data not shown). The poor differentiation of SCC is independently associated with local recurrence, lymph node metastasis, and disease-specific death (30). The difference in TLR4 expression may, therefore, be associated with unfavorable outcomes in poorly differentiated SCC. However, in this study, the number of patients with TLR4 staining of AK, $\mathrm{BD}$, and poorly differentiated $\mathrm{SCC}$, as well as the number of patients with CD44 staining of SCC was small. Thus, further clinicopathological studies using a greater number of cases are required to confirm the biological role of TLR4 and CD44 in skin tumors.

An interaction between TLR4 and CD44 was previously demonstrated in breast cancer cells (24). In cutaneous SCC, Karvinen et al reported that poorly-differentiated SCC tumors showed an irregular CD44 staining pattern and reduced expression with areas of missing or low intensity (19); conversely, well-differentiated SCC exhibited homogeneous CD44 staining and moderate intensity (19). Although the interaction between TLR4 and CD44 in cutaneous cancers is unclear, these reports and the findings of this study suggest that an irregular CD44 expression and localization may be associated with a perturbed TLR4 expression and localization in poorly differentiated SCC.

In this study, we analyzed the biological role of TLR4 and the association between TLR4 and CD44 in cutaneous SCC cells. The knockdown of TLR4 expression by siRNA accelerated cell migration and invasion compared to the control siRNA-transfected HSC-1 and HaCaT cells (Fig. 5). Notably, TLR4 expression in the TLR4 siRNA-transfected HSC-1 cells was mainly reduced in the cytoplasm, and to a certain degree, TLR4 expression remained detectable in the cell membrane (Fig. 7A, panels a and d). A similar TLR4 expression and localization pattern was observed in the poorly differentiated SCC tissues (Fig. 2A, panel b). Furthermore, the knockdown of TLR4 increased CD44 expression (Figs. 6, 7B and 8B) and 

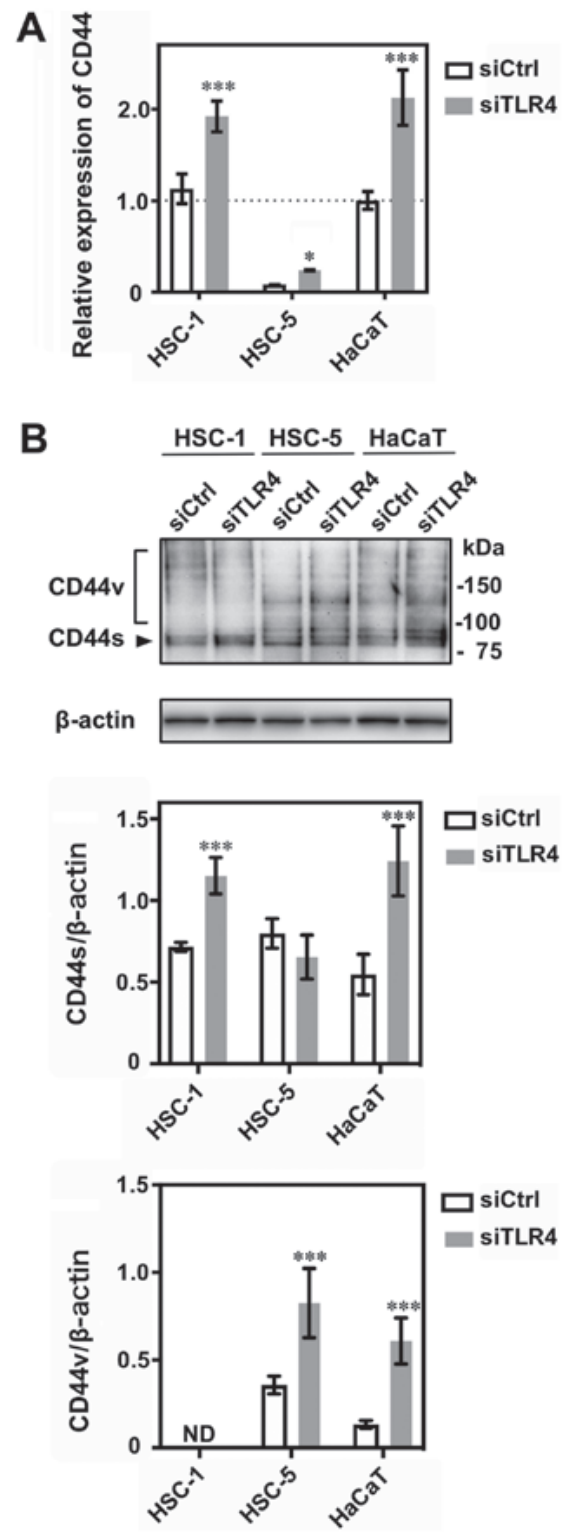

Figure 6. CD44 mRNA and protein expression in siTLR4-transfected cells (A) The relative expression of CD44 mRNA was analyzed by RT-qPCR following transfection of the HSC-1, HSC-5 and HaCaT cells with siTLR4. The relative expression of CD44 mRNA is indicated as a percentage of that of siCtrl-treated $\mathrm{HaCaT}$ cells. The data represent the means $\pm 95 \%$ confidence interval of triplicate measurements in 3 independent experiments. Statistical significance between siTLR 4 or siCtrl groups and among cell lines was determined by two-way ANOVA followed by Sidak's multiple comparisons test. HSC-1: ${ }^{* * *} \mathrm{P}<0.001$; HSC-5: $\mathrm{P}=0.0358$; HaCaT: ${ }^{* * * *} \mathrm{P}<0.001$. (B) Western blot analysis of $\mathrm{CD} 44$ protein expression following transfection with siTLR4 in HSC-1, HSC-5 and HaCaT cells. The data represent the mean $\pm 95 \%$ confidence interval of triplicate measurements in 3 independent experiments. Statistical significance between siTLR4 or siCtrl groups and among cell lines was determined by two-way ANOVA followed by Sidak's multiple comparisons test. CD44s/ $\beta$-actin: HSC- $1,{ }^{* * *} \mathrm{P}<0.001$; HSC-5, $\mathrm{P}=0.2111$; HaCaT, ${ }^{* * * *} \mathrm{P}<0.001$. CD44v/ $\beta$-actin: HSC-1, not detected (ND); HSC-5, ${ }^{* * *} \mathrm{P}<0.001$; HaCaT, ${ }^{* * *} \mathrm{P}<0.001$. CD44s: CD44 standard form, CD44v: CD44 variant isoforms (140 kDa). TLR4, Toll-like receptor 4; SCC, squamous cell carcinoma; siTLR4, siRNA against TLR4; siCtrl, control siRNA.

the filopodia protrusions formation (Fig. 7A, panels d-f and B, panels d-f). Filopodia are thin, finger-like membrane protrusions that extend out from the cell edge and are involved in cell migration and cell invasion (31). These results suggest that reduced TLR4 expression may enhance the malignant
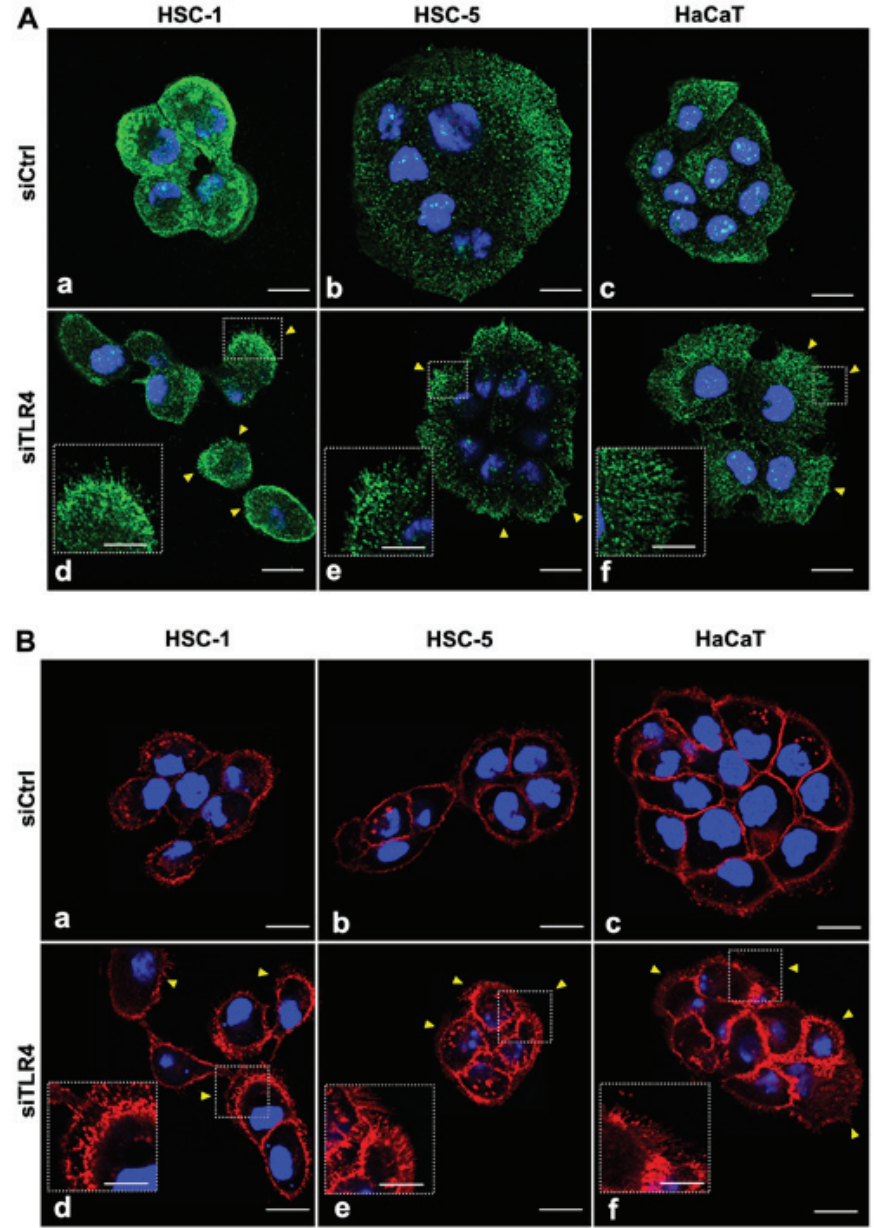

Figure 7. Immunofluorescence staining of TLR4 and CD44 in siTLR4-transfected cells. HSC-1, HSC-5 and HaCaT cells were transfected with siCtrl (panels a, b and c, respectively) or siTLR4 (panels d, e and f, respectively), and then stained with a (A) TLR4 or (B) CD44 antibody. The nuclei were stained with DAPI (blue). (A) TLR4 knockdown in HSC-1 cells decreased TLR4 expression (green) in the cytoplasm and enhanced the formation of filopodia protrusions (arrowheads) compared to siCtrl cells. (B) TLR4 knockdown increased CD44 expression (red) in the cell membrane and increase the formation of filopodia protrusions (arrowheads) compared to siCtrl cells. The insets show a high-power view of filopodia protrusions. All images show a single optical section. Scale bar, $20 \mu \mathrm{m}$. TLR4, Toll-like receptor 4; SCC, squamous cell carcinoma; siTLR4, siRNA against TLR4; siCtrl, control siRNA.

features of cutaneous SCC. In the future, we aim to focus on investigating the role of TLR4 in epithelial-mesenchymal transition, which is deeply implicated in cancer cell migration and invasiveness.

In this study, the TLR4 protein levels appeared to still be high in the TLR4 siRNA-transfected HSC-5 cells, while the mRNA levels were significantly decreased. However, the lack of the successful knockdown of TLR4 protein in HSC-5 cells may have affected the cell migration and CD44 expression results. These results may be due to cell biological differences between HSC-1 and HSC-5 cells, such as differences in stability between mRNA and protein, or difference in protein degradation processes.

HSC-1 is a cell line derived from poorly differentiated human skin SCC on the hand (26). HSC-5 is a cell line derived from well-differentiated human skin SCC on the auricle (27). However, the differential ability of keratinization 

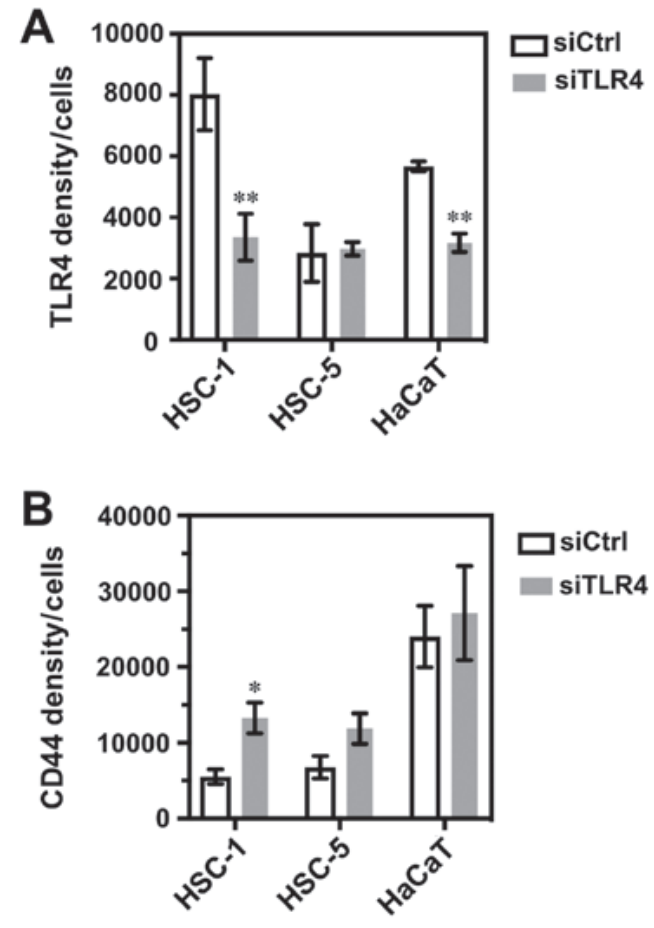

Figure 8. Quantitative evaluation of Immunofluorescence staining. The analysis of the fluorescence signal of TLR4 and CD44 was carried out on 5 randomly selected fields per X20 objective sample. (A) TLR4 integrated density/cells: HSC-1, ${ }^{* *} \mathrm{P}=0.0077$; HSC-5, $\mathrm{P}=0.6873$; HaCaT, ${ }^{* *} \mathrm{P}=0.0073$. (B) CD44 integrated intensity/cells: HSC-1, ${ }^{*} \mathrm{P}=0.0177$; HSC-5, $\mathrm{P}=0.1099$; $\mathrm{HaCaT}, \mathrm{P}=0.6683$. The data represent the means \pm SEM of triplicate measurements in three independent experiments. Statistical significance between siTLR4 or siCtrl groups and among cell lines was determined by multiple test using the Holm-Sidak method. TLR4, Toll-like receptor 4; SCC, squamous cell carcinoma; siTLR4, siRNA against TLR4; siCtrl, control siRNA.

was lacking in both cell lines, and the patterns of expression levels of cytokeratin proteins of HSC-1 and HSC-5 differed. In addition, the HSC-1 cells were grown and they exhibited features of keratinization, such as horn peals and individual cell keratinization in a xenograft mouse model (26), while HSC-5 transplantation was not successful (27). The difference in reactivity to TLR4 siRNA between the HSC-1 and HSC-5 cells may be caused by these cell biological differences. Additional work using other SCC cell lines will provide more evidence to support our results.

TLR4 may exert anti-tumor or pro-tumor effects. However, a number of studies have described a pro-tumor role of TLR4 expression in cancer cells $(4-6,13,32,33)$. In addition, a dual role of TLR4 in breast cancer cells has been identified: TLR4 activation inhibits TP53 wild-type cell growth but promotes TP53 mutant cell growth by regulating proliferation (34). Notably, the findings of this study indicate an anti-tumor role of TLR4 expression in SCC cells. This may be explained by the increased CD44 expression in response to the decreased TLR4 expression.

CD44 is a transmembrane glycoprotein that is highly expressed in a number of types of cancer and cancer stem cells. CD44 interacts with several extracellular matrix ligands, including hyaluronan or hyaluronic acid, osteopontin, collagen and fibronectin to induce actin cytoskeleton regulation, cell migration and invasion $(22,35)$. CD44s and CD44v have overlapping, yet distinct roles in cancer cell proliferation, adhesion, migration and invasion $(22,35)$. In this study, we found that CD44s expression increased in the TLR4 siRNA-transfected HSC-1 and HaCaT cells. The CD44v isoform was also expressed in the HSC-5 and $\mathrm{HaCaT}$ cells and was similarly enhanced following TLR4 siRNA transfection (Fig. 6B). These findings indicate that both CD44s and CD44v expression may contribute to cell migration and invasive ability in cutaneous SCC, depending on the cell types. Only a few studies to date have examined the association between TLR4 and CD44 in cancer cells, at least to the best of our knowledge. Bourguignon et al reported that low molecular weight hyaluronan stimulated the association between CD44 and TLRs (TLR2 and 4), followed by concomitant recruitment of AFAP-110 and MyD88 that promotes tumor-cell invasion in breast tumor cells (24). We suggest a possibility that TLR4 and CD44 may be associated through a negative feedback mechanism in cutaneous SCC. Overexpression experiments for TLR4 will be required to support this hypothesis.

To the best of our knowledge, this is the first study to report an association between TLR4 and CD44 that contributes to tumor migration and invasion in cutaneous SCC. We found that decreased TLR4 expression levels are associated with enhanced malignant features in human SCC tissue samples and cultured SCC cell line. TLR4 may thus play an important anti-tumor role in suppressing aggressive cutaneous SCC cellular behaviors.

\section{Acknowledgements}

Not applicable.

\section{Funding}

This study was supported, in part, by Grants-in-Aid for the Clinical Rebiopsy Bank Project for Comprehensive Cancer Therapy development to ZN from the Ministry of Education, Culture, Sports, Science and Technology, Japan (MEXT), 2013-2017 (S13110022) and a Grant-in-Aid for scientific research (C, no. 16K00406 to MK) from JSPS KAKENHI.

\section{Availability of data and materials}

The datasets used and analyzed during the current study are available from the corresponding author on reasonable request.

\section{Authors' contributions}

MK and ZN participated in the design of the study. EM and RO performed the histological examination and analysis. EM, KK, YK, KT, TF, and TK participated in data collection and performed research. EM wrote the manuscript, and MK, RO, SK, KI, TS, RW, HS, and ZN critically revised the manuscript and participated in the analysis and interpretation of the data. All authors have reviewed, edited, and approved the final version of the manuscript.

\section{Ethics approval and consent to participate}

The Nippon Medical School Hospital Institutional Review Board approved this study (approval no. 29-07-788, August 18th, 2017) and written informed consent was obtained from all patients. 


\section{Patient consent for publication}

All participants provided written informed consent for the study.

\section{Competing interests}

The authors have no competing interests with respect to this study.

\section{References}

1. Burton KA, Ashack KA and Khachemoune A: Cutaneous Squamous Cell Carcinoma: A Review of High-Risk and Metastatic Disease. Am J Clin Dermatol 17: 491-508, 2016.

2. Karia PS, Han J and Schmults CD: Cutaneous squamous cell carcinoma: Estimated incidence of disease, nodal metastasis, and deaths from disease in the United States, 2012. J Am Acad Dermatol 68: 957-966, 2013

3. Zhou M, McFarland-Mancini MM, Funk HM, Husseinzadeh N, Mounajjed T and Drew AF: Toll-like receptor expression in normal ovary and ovarian tumors. Cancer Immunol Immunother 58: 1375-1385, 2009.

4. Sun Y, Wu C, Ma J, Yang Y, Man X, Wu H and Li S: Toll-like receptor 4 promotes angiogenesis in pancreatic cancer via PI3K/AKT signaling. Exp Cell Res 347: 274-282, 2016.

5. Dong YQ, Lu CW, Zhang L, Yang J, Hameed W and Chen W: Toll-like receptor 4 signaling promotes invasion of hepatocellular carcinoma cells through MKK4/JNK pathway. Mol Immunol 68 : 671-683, 2015.

6. Ye K, Wu Y, Sun Y, Lin J and Xu J: TLR4 siRNA inhibits proliferation and invasion in colorectal cancer cells by downregulating ACAT1 expression. Life Sci 155: 133-139, 2016.

7. Zou Y, Qin F, Chen J, Meng J, Wei L, Wu C, Zhang Q, Wei D, Chen X, Wu H, et al: sTLR4/MD-2 complex inhibits colorectal cancer in vitro and in vivo by targeting LPS. Oncotarget 7: 52032-52044, 2016.

8. Eiró N, Ovies C, Fernandez-Garcia B, Álvarez-Cuesta CC, González L, González LO and Vizoso FJ: Expression of TLR3, 4, 7 and 9 in cutaneous malignant melanoma: Relationship with clinicopathological characteristics and prognosis. Arch Dermatol Res 305: 59-67, 2013.

9. Janda J, Burkett NB, Blohm-Mangone K, Huang V, Curiel-Lewandrowski C, Alberts DS, Petricoin EFIII, Calvert VS, Einspahr J, Dong Z, et al: Resatorvid-based Pharmacological Antagonism of Cutaneous TLR4 Blocks UV-induced NF- $x \mathrm{~B}$ and AP-1 Signaling in Keratinocytes and Mouse Skin. Photochem Photobiol 92: 816-825, 2016.

10. Blohm-Mangone K, Burkett NB, Tahsin S, Myrdal PB, Aodah A, Ho B, Janda J, McComas M, Saboda K, Roe DJ, et al: Pharmacological TLR4 Antagonism Using Topical Resatorvid Blocks Solar UV-Induced Skin Tumorigenesis in SKH-1 Mice. Cancer Prev Res (Phila) 11: 265-278, 2018.

11. Iotzova-Weiss G, Freiberger SN, Johansen P, Kamarachev J, Guenova E, Dziunycz PJ, Roux GA, Neu J and Hofbauer GFL: TLR4 as a negative regulator of keratinocyte proliferation. PLoS One 12: e0185668, 2017.

12. Szatmary Z: Molecular biology of toll-like receptors. Gen Physiol Biophys 31: 357-366, 2012.

13. Mai CW, Kang YB and Pichika MR: Should a Toll-like receptor 4 (TLR-4) agonist or antagonist be designed to treat cancer? TLR-4: Its expression and effects in the ten most common cancers. Onco Targets Ther 6: 1573-1587, 2013.

14. Dajon M, Iribarren K and Cremer I: Toll-like receptor stimulation in cancer: A pro- and anti-tumor double-edged sword. Immunobiology 222: 89-100, 2017.

15. Miller LS: Toll-like receptors in skin. Adv Dermatol 24: 71-87, 2008.
16. Weng H, Deng Y, Xie Y, Liu H and Gong F: Expression and significance of HMGB1, TLR4 and NF- $\mathrm{BB}$ p65 in human epidermal tumors. BMC Cancer 13: 311, 2013.

17. Burns EM and Yusuf N: Toll-like receptors and skin cancer. Front Immunol 5: 135, 2014.

18. Yusuf N, Nasti TH, Long JA, Naseemuddin M, Lucas AP, Xu H and Elmets CA: Protective role of Toll-like receptor 4 during the initiation stage of cutaneous chemical carcinogenesis. Cancer Res 68: 615-622, 2008.

19. Karvinen S, Kosma V-MM, Tammi MII and Tammi R: Hyaluronan, CD44 and versican in epidermal keratinocyte tumours. Br J Dermatol 148: 86-94, 2003.

20. Hartmann-Petersen S, Tammi RH, Tammi MI and Kosma VM: Depletion of cell surface CD44 in nonmelanoma skin tumours is associated with increased expression of matrix metalloproteinase 7. Br J Dermatol 160: 1251-1257, 2009.

21. Erfani E, Roudi R, Rakhshan A, Sabet MN, Shariftabrizi A and Madjd Z: Comparative expression analysis of putative cancer stem cell markers CD44 and ALDH1A1 in various skin cancer subtypes. Int J Biol Markers 31: e53-e61, 2016.

22. Senbanjo LT and Chellaiah MA: CD44: A Multifunctional Cell Surface Adhesion Receptor Is a Regulator of Progression and Metastasis of Cancer Cells. Front Cell Dev Biol 5: 18, 2017.

23. Bourguignon LYW: Matrix hyaluronan-activated CD44 signaling promotes keratinocyte activities and improves abnormal epidermal functions. Am J Pathol 184: 1912-1919, 2014.

24. Bourguignon LYW, Wong G, Earle CA and Xia W: Interaction of low molecular weight hyaluronan with CD44 and toll-like receptors promotes the actin filament-associated protein 110 -actin binding and MyD88-NFאB signaling leading to proinflammatory cytokine/chemokine production and breast tumor invasion. Cytoskeleton (Hoboken) 68: 671-693, 2011.

25. Elder DE, Massi D and Scolyer R and Willemze R (eds): WHO Classification of Skin Tumours. Fourth Edition. In: WHO Classification of Tumours. Vol 11. IARC, Lyon, 2018.

26. Kondo S and Aso K: Establishment of a cell line of human skin squamous cell carcinoma in vitro. Br J Dermatol 105: 125-132, 1981.

27. Hozumi Y, Kondo S, Shimoura T and Aso K: Human squamous cell carcinoma from skin: Establishment and characterization of a new cell line (HSC-5). J Dermatol 17: 143-148, 1990.

28. Livak KJ and Schmittgen TD: Analysis of relative gene expression data using real-time quantitative PCR and the 2(-Delta Delta C(T)) Method. Methods 25: 402-408, 2001.

29. Yanofsky VR, Mercer SE and Phelps RG: Histopathological variants of cutaneous squamous cell carcinoma: A review. J Skin Cancer 2011: 210813, 2011.

30. Motaparthi K, Kapil JP and Velazquez EF: Cutaneous Squamous Cell Carcinoma: Review of the Eighth Edition of the American Joint Committee on Cancer Staging Guidelines, Prognostic Factors, and Histopathologic Variants. Adv Anat Pathol 24: 171-194, 2017.

31. Jacquemet G, Hamidi $\mathrm{H}$ and Ivaska J: Filopodia in cell adhesion, 3D migration and cancer cell invasion. Curr Opin Cell Biol 36: 23-31, 2015.

32. Dang S, Peng Y, Ye L, Wang Y, Qian Z, Chen Y, Wang X, Lin Y, Zhang X, Sun X, et al: Stimulation of TLR4 by LMW-HA induces metastasis in human papillary thyroid carcinoma through CXCR7. Clin Dev Immunol 2013: 712561, 2013.

33. Yu L, Wang L and Chen S: Dual character of Toll-like receptor signaling: Pro-tumorigenic effects and anti-tumor functions. Biochim Biophys Acta 1835: 144-154, 2013.

34. Haricharan S and Brown P: TLR4 has a TP53-dependent dual role in regulating breast cancer cell growth. Proc Natl Acad Sci USA 112: E3216-E3225, 2015.

35. Chen C, Zhao S, Karnad A and Freeman JW: The biology and role of CD44 in cancer progression: Therapeutic implications. J Hematol Oncol 11: 64, 2018.

36. Brierley JD, Gospodarowicz MK and Wittekind C (eds): TNM Classification of Malignant Tumours. 8th edition. Union for International Cancer Control (UICC), Geneva, 2016. 